\title{
DEFLECTING RF CAVITY DESIGN FOR A RECIRCULATING LINAC BASED FACILITY FOR ULTRAFAST X-RAY SCIENCE (LUX)*
}

\author{
Derun $\mathrm{Li}^{\#}$ and J. N. Corlett \\ Lawrence Berkeley National Laboratory, Berkeley, CA 94720, USA
}

\section{Abstract}

We report on superconducting deflecting RF cavity designs for a Recirculating Linac Based Facility for Ultrafast $\mathbf{X}$-ray Science (LUX) at Lawrence Berkeley National Laboratory. The deflecting cavities operate in the lowest dipole mode and are required to produce a temporal correlation within flat electron bunches, as needed for x-ray compression in crystal optics. Deflecting voltage of up to $8.5-\mathrm{MV}$ is required at $3.9-\mathrm{GHz}$. We present a 7-cell cavity design in this paper. Seven such cavities are required to generate the 8.5 MV deflecting voltage. Longitudinal and transverse impedance from LOM (lower order mode) and HOM (higher order mode) are simulated using the MAFIA code. Short-range and long-range wakefield excited through these impedances are calculated. Beam loading effects of the deflecting mode and LOM modes are estimated. $Q$ values of the LOM monopole modes in the cavity may need to be damped to be below $10^{4}-10^{5}$ levels in order to maintain the required energy spread.

\section{INTRODUCTION}

Figure is a layout of the LUX machine at LBNL. The SC deflecting cavities (highlighted with yellow color) are required at the last turn of the flat electron bunch at energy of $\sim 3-\mathrm{GeV}$. More information on LUX machine can be found in [1].

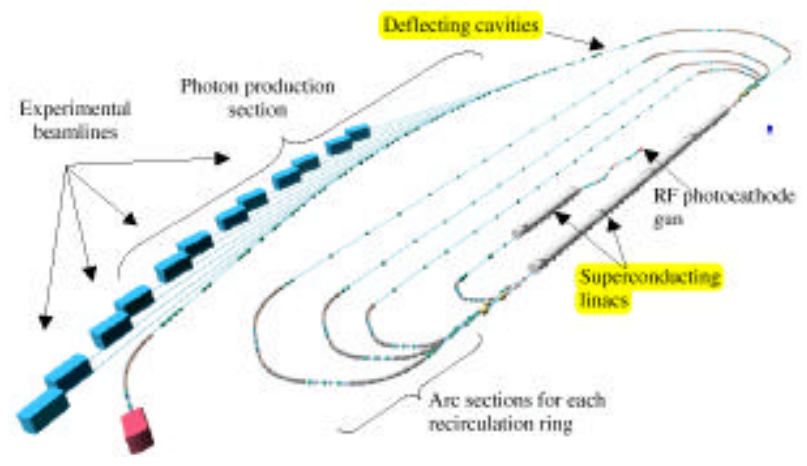

Figure 1: The layout of the proposed LUX at LBNL

RF cavities operating in the lowest dipole mode $\left(\mathrm{TM}_{110^{-}}\right.$ like) deflect the head and tail of $\sim 3 \mathrm{GeV}$ flat electron bunches to allow for compression of the x-ray pulse in beam line optics. The center of the electron bunch passes the cavities at zero phase of the RF field such that the head and tail are deflected in opposite directions, and the center of the bunch experiences no deflection. This introduces a divergence into the electrons within a bunch that is much greater than the opening angle of hard x-ray radiation (the radiation opening angle is $7 \mu \mathrm{rad}$ at $1 \AA$ ), and the angular divergence of the electrons $(\sim 6.4 \mu \mathrm{m})$ in the undulators. The required deflecting voltage is $8 \mathrm{MV}$ at $3.9 \mathrm{GHz}$ for $\sim 3 \mathrm{GeV}$ beam energy, but we aim the cavity design for 8.5 MV. Superconducting cavities are a natural choice in obtaining such a high voltage, and the design of a multi-cell structure with large transverse shunt impedance has been developed. A seven-cell $\pi$-mode cavity design has been selected. This choice represents a compromise between a large number of cells to increase transverse shunt impedance and reduce the number of cavities required, and smaller number of cells to minimize the number of cavity modes and potential mode coupling. The cavity design is similar to a multi-cell deflecting cavity design for the kaon separation project at Fermilab [2].

\section{THE DEFLECTING CAVITY}

The deflecting cavity operates at the lowest dipole mode, or call $\mathrm{TM}_{110}$-like mode. Field distribution of this mode is shown in Figure 2 using a single cylindrical pillbox beam pipe as an example.

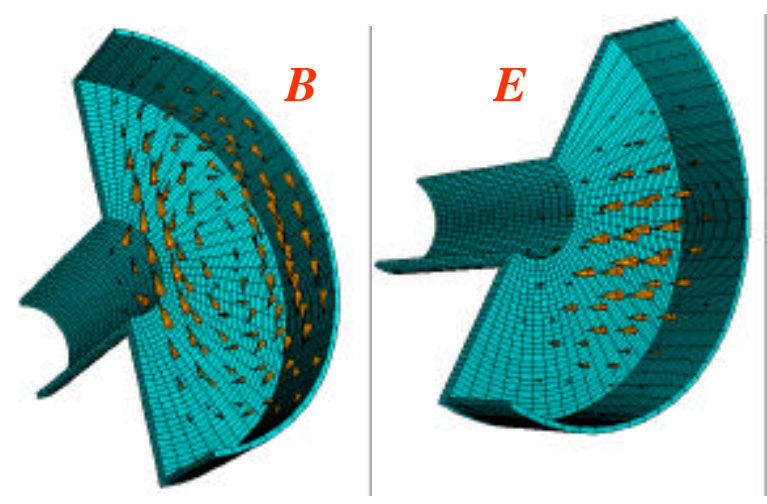

Figure 2: Electrical (right) and magnetic field distribution of the lowest dipole mode in a cylindrical pillbox cavity. This mode is used for deflect electron bunches to generate a temporal correlation for $\mathrm{x}$-ray compression.

For cylindrical cavities, dipole modes are degenerate, and they have the same field distribution, but with different orientations. In order to obtain the needed polarization, the cavity geometry needs to be perturbed in one plane. Figure 3 shows a KEK-B crab cavity with one plane being squashed [4]. Fermilab multi-cell deflecting cavity used the similar technique.

\footnotetext{
* Work supported by the US Department of Energy under contract No. DE-AC0376SF00098

\#Email: Dli@1bl.gov
} 


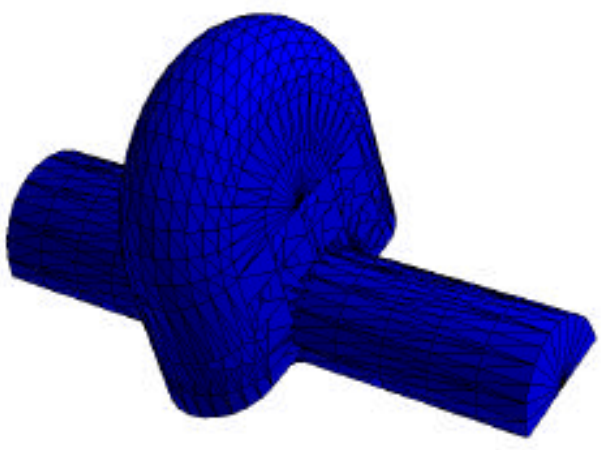

Figure 3: Squashed shape KEK-B Crab cavity in order to obtain the polarization of the crabbing mode

Electron bunches passing through the deflecting cavity experience transverse forces from both electric and magnetic fields. The cavity shunt impedance definition then has to take this into account.

\section{Shunt Impedance of the Dipole Mode}

For the TM dipole mode of an ideal closed cylindrical pillbox cavity, there is no electric field on-axis. Beam passing through such a cavity on-axis would experience a transverse force from the magnetic fields only. However once beam irises are introduced, as is necessary for a practical cavity, TE-like modes are introduced and mixed with TM modes in the iris (or between cells) and beampipe regions. The deflecting mode is no longer a pure $\mathrm{TM}_{110}$, but a hybrid of $\mathrm{TM}_{110}$ and $\mathrm{TE}_{111}$ modes, which is the result of Maxwell equations in order to satisfy the new boundary conditions introduced by the irises and beampipes. Even for the beam traversing the cavity on-axis will not just experience transverse force from the magnetic fields, but also from the transverse electric field. For a cavity with a $\pi$ phase advance, these two transverse forces add up. To calculate the shunt impedance of the deflecting mode, we define the transverse shunt impedance as follows,

$$
\left(\frac{R}{Q}\right)_{\perp}=\frac{\left|\int E_{Z}\left(r_{0}\right) e^{j \kappa z} d z\right|^{2}}{\omega U\left(\kappa r_{0}\right)^{2}}
$$

Where the Panofsky-Wenzel theorem is applied to obtain the deflecting voltage using the off-axis longitudinal electric fields only. $V_{\perp}$ is the transverse voltage; $\omega=2 \pi f$ with $f$ as the resonant frequency; $U$ the stored energy of the mode at the resonant frequency; $E\left(r_{0}\right)$ the longitudinal electric field at $r_{0}$ over which the longitudinal electric field is integrated along variable $z$. Note that the definition of transverse shunt impedance is in $\Omega$, and implies that the deflecting kick is independent of beam's transverse position within the iris.

\section{Design of the Multi-Cell Cavity}

A 3-D MAFIA model, as shown in Figure 4, was established to simulate the deflecting cavity and the main RF coupler in combination with 2-D simulations reported before [3]. 7-cell is a choice in consideration of field flatness, mode overlaps and possible trapped modes resulted from large number of cells.

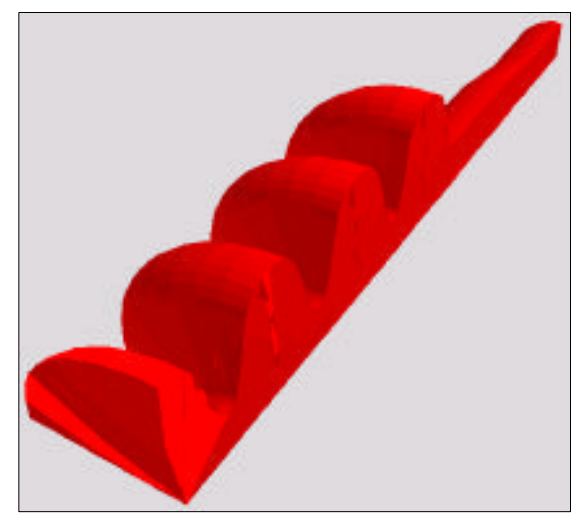

Figure 4: 3-D MAFIA model for the 7-cell deflecting cavity (1/4 of the structure was shown here)

One-quarter of the cavity was used for the simulation, combination between boundary conditions and geometry symmetry allow us to compute all LOM and HOM modes up to beam-pipe cut-off frequencies. Table 1 and Table 2 give the dimensions of cavity and parameters of expected performance.

Table 1: Main dimensions of the deflecting cavity

\begin{tabular}{|l|c|c|}
\hline Cavity frequency & 3.9 & $\mathrm{GHz}$ \\
\hline Phase Advance per cell & $180^{\circ}$ & Degree \\
\hline Cavity Equator Curvature & 1.027 & $\mathrm{~cm}$ \\
\hline Cavity Radius & 4.795 & $\mathrm{~cm}$ \\
\hline Cell length & 3.846 & $\mathrm{~cm}$ \\
\hline Iris Radius & 1.500 & $\mathrm{~cm}$ \\
\hline Beam pipe radius & 1.500 & $\mathrm{~cm}$ \\
\hline TM mode cut-off frequency & 7.634 & $\mathrm{GHz}$ \\
\hline TE mode cut-off frequency & 5.865 & $\mathrm{GHz}$ \\
\hline
\end{tabular}

Table 2: Main cavity parameters

\begin{tabular}{|c|c|c|}
\hline$(\mathrm{R} / \mathrm{Q})$ & 350 & $\Omega$ \\
\hline $\mathrm{Q}_{0}$ & $2 \times 10^{9}$ & \\
\hline Active length/cavity & 26.92 & $\mathrm{~cm}$ \\
\hline Deflecting gradient & 5 & $\mathrm{MV} / \mathrm{m}$ \\
\hline Transverse voltage & 1.346 & $\mathrm{MV}$ \\
\hline RF power loss at $2 \mathrm{~K}$ & 2.6 & Watts \\
\hline
\end{tabular}


The $5-\mathrm{MV} / \mathrm{m}$ gradient was determined by limiting critical magnetic fields to be $\sim 80 \mathrm{mT}$, which corresponds to a $25 \mathrm{MV} / \mathrm{m}$ accelerating gradient for the TESLA SC cavities.

\section{BEAM LOADING}

Beam traversing the deflecting cavity interacts with the cavity through coupling impedances, beam may lose energy, and induce a voltage in the cavity. This voltage then acts back on the beam and may cause energy spread or perturb the deflection along the bunch.

\section{Beam Loading in the Deflecting Mode}

The beam induced transverse voltage in the deflecting mode may be written as,

$$
V_{\perp}=\left(\frac{R}{Q}\right)_{\perp} \frac{Q_{0}}{1+\beta}(\kappa \Delta r) I \approx 1.1 \mathrm{kV}(\sim 0.09 \% \mathrm{f} 1.3 \mathrm{MV})
$$

Where $I, k, \Delta r$ and $\beta$ are beam current, wave number, displacement from cavity axis and RF coupling constant, respectively. At $3.9 \mathrm{GHz}, \kappa=82, \Delta r=0.1 \mathrm{~mm}$ and $\beta \approx$ 50 assuming an achievable bandwidth of $100 \mathrm{~Hz}$ which corresponds to an external $Q$ of $3.9 \times 10^{7}$. This bandwidth is determined from the requirements for synchronization, microphonics (25 Hz peak), and 2.5 MHz band limited phase noise spectra of mode-locked laser master oscillator at optimum coupling condition. The peak RF power required is less than 200 watts per 7-cell cavity. Beam current of $10 \mu \mathrm{A}(1 \mathrm{nC}$ charge at $10 \mathrm{kHz}$ repetition rate) was assumed for the above calculations.

Power induced in the deflecting mode is then given by,

$$
P_{\text {induced }}=\frac{1}{2} f_{\text {rep. }}^{2} q^{2}\left(\frac{R}{Q}\right)_{\perp}^{*} \frac{Q_{0}}{1+\beta}(\kappa \Delta r) \approx 5.3 \mathrm{~mW}
$$

This is considered to be quite small in comparison with power loss of 2.6 watts in the cavity.

\section{Beam Loading from Monopole Modes}

Longitudinal voltages may be induced by beam through interaction with monopole LOM and HOM modes. In fact two LOM monopole modes at $2.8581 \mathrm{GHz}$ and 2.8685 $\mathrm{GHz}$ contribute $84 \%$ of the total impedance. At steady state the beam induced voltage per cavity can be calculated by,

In order to maintain beam energy spread of $10^{-4}$, these modes need to be damped. Their $Q$ values have to be

$V(\infty)=\sum_{n=1}^{n_{c}} 2 k_{n} q\left(\frac{2 Q_{n, 0}}{\omega_{n} T}\right) \approx 17 \mathrm{MV}$

Assuming $q=1 \mathrm{nC}, 10 \mathrm{kHz}$ repetition rate and $Q_{0}=2 \times 10^{9}$ for all the modes below $10^{4}-10^{5}$ levels. Main RF coupler may provide certain damping to all the HOM and LOM modes, but has not been evaluated yet. Nevertheless a coaxial insert in the beam pipe (proposed for KEK-B crab cavity) should couple to monopole modes only and provide strong damping to monopole modes.

\section{HOM Dipole Modes}

Loss factors of HOM dipole modes were calculated by,

$$
k_{\perp}=\frac{\omega}{4}\left(\frac{R}{Q}\right)_{\perp} \mathbf{K} .
$$

Again assuming $2 \times 10^{9}$ of $Q$ for all HOM modes, total loss fact is only $14 \mathrm{~V} /(\mathrm{pC}-\mathrm{m})$, compared to $176 \mathrm{~V} / \mathrm{pC}-\mathrm{m})$ of the deflecting mode. The overall HOM impedance is considered to be not excessive.

\section{CONCLUSION}

A 7-cell SC deflecting cavity design has been presented. To provide 8.5 MV deflecting voltage, seven 7-cell cavities are required. LOM monopole modes need to be damped to a level that $10^{-4}$ beam energy spread can be maintained. Impedance from the HOM dipole modes is not excessive. Main RF coupler may provide some damping to LOM and HOM modes and will be evaluated in the future. RF power requirement is estimated to be less than 200 watts per 7-cell cavity at optimum coupling. Solid state RF power amplifier may be used at this power level. Synchronization requirement may demand for even wider cavity band-with, but will be investigated further with the development of the project.

\section{REFERENCES}

[1] J. Corlett, et al., "A Recirculating Linac-Based Facility for Ultrfast X-Ray Science, this conference.

[2] L. Bellantoni, et al., "Design and Measurements of a Deflecting Mode Cavity for an RF Separator", PAC 2001, Chicago, IL, USA

[3] D. Li and J. Corlett, "RF Deflecting Cavity Design for Berkeley Ultrafast X-Ray Source”, EAPC 2002, Paris, France

[4] K. Akai, "Development of Crab Cavity for CESR-B", Proceedings of PAC 1993, USA 